\title{
Fluid Flow Analysis of a New Finned Piston Reciprocating Compressor Using Pneumatic Analogy
}

\author{
Mahbod Heidari and Alfred Rufer
}

\begin{abstract}
The goal of this paper is to investigate the fluid flow analysis in a novel multilayered finned reciprocating compressor, adopting a so-called "pneumatic-Electric" analogy using a lumped methodology. This machine includes two sets of concentric annular fins with different diameters: the mobile fins are pushed into the space between the stationary fins through a driver shaft and compress the air trapped in the interconnecting annular chambers. Considering pressure as effort and mass flow as the flow variable, the equivalent electric circuit was developed to represent the behavior of the pneumatic system. The governing state space equations are derived and simulation results are presented.
\end{abstract}

Index Terms-Pneumatic, electric, pressure, mass flow.

\section{INTRODUCTION}

Hydraulics analogy has been used widely to model and control hydraulic systems [1]-[5]. However, analogy between pneumatic systems to electrical circuits is rare in publications. This analogy is of great interest in systems with compressible - or incompressible - flow as working fluid and with high complexity and numerous similar or axisymmetric chambers, such as compressors and gas turbines. The finned compressor under study is interesting to be analyzed using such an analogy since it can be divided into similar subsystems. In the first section the compressor system will be explained and its geometry and its movement principle in one cycle will be described.

Then in second section the principles of pneumatic analogy will be reminded and equivalent terms for capacitor, resistance and inductor will be derived.

Third part of the paper will construct a circuit for a classic piston with one chamber. The analogy will be expanded to the fined piston in Section IV.

\section{SYSTEM DESCRIPTION}

To solve the problems of non-isothermal compression in piston compressors, a new concept has been proposed recently in LEI Laboratory at EPFL, called "Finned Piston". In this design, a series of concentric metal annuli's are used to fit into each other to compress the air (Fig. 1). As a result, the heat exchange area increases dramatically which increases the heat transfer leading to higher isothermal efficiency.

In such a compressor, one mobile assembly of differential

Manuscript received February 4, 2014; revised April 3, 2014. This work was supported in part by European union through Solution project.

M. Heidari is with the École Polytechnique Fédérale de Lausanne (EPFL), Lausanne, 1015, Switzerland (e-mail: mahbod.heidari@ epfl.ch).

A. Rufer is with the Laboratoire d'Électronique Industrielle (LEI) at EPFL lausanne, Switzerland. layers is sliding inside of a fixed assembly of fins. In the center of the cylinder, there is a shaft for guiding the mobile equipment inside the fixed one, and that allows designing the system with a small distance between the fins.

The parts numbered on the figure are:

1) External cylinder

2) Stator fins

3) Sliding fins

4) Guiding rod

5) Stator seal (rod-seal)

6) Piston-seal

7) Gas Inlet/outlet

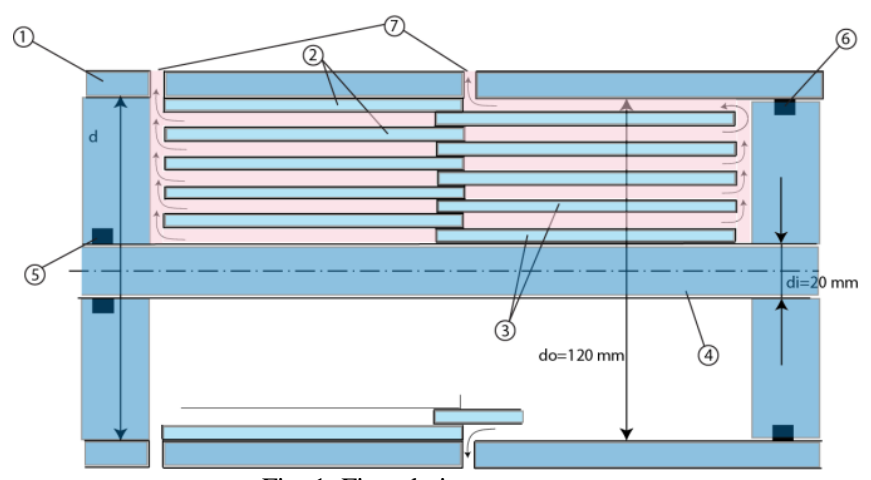

Fig. 1. Finned piston geometry.

The inlet/outlet of the compressed/expanded gas in such a concept is realized through radial paths, allowing the access to the internal layers. The radial paths can be arranged on both sides of the cylinder, eventually at the whole periphery.

On the left side of Fig. 1, the radial in/outlet is shown, and corresponds to the access to the annular compression/expansion chambers of the fixed (stator) part. In the middle of the figure, the radial in/outlet path is represented for the mobile (piston-side) annular chambers. It is evident that the two paths must be connected to the same in/outlet circuit or valve, using a manifold collector.

\section{Pneumatic Analogy}

Modeling a reciprocating compressor deals with a series of complicated phenomena's occurring simultaneously in a relative short period of time. Authors have shown in another article [6], that this can be best done by detaching pressure-mass flow as one pair (pneumatic bond) and temperature-heat transfer (thermal bond) and dealing with them separately. However these parameter sets can not be fully detached since they are correlated by constitutive relations and energy and mass conservation equations, but by doing so the model can be handles easier. In this paper the pneumatic analogy will be discussed. Thermoelectricity analogy (thermal bond) is presented by authors in another publication [7]. 
It is common practice in hydraulics to choose pressure as effort (or potential) and volume flow as the flow variable.

In pneumatics or gases however, since the density is not constant and the flow can be regarded as compressible, mass flow is considered as the flow variable.

Hagen-Poiseuille law indicates that pressure drop and mass flow rate are related as [8]:

$$
\Delta p=\frac{128 \mu l}{\rho \pi d^{4}} \dot{m}
$$

Comparing (1) to Ohm's law indicates that resistance in pneumatic systems is proportional to the length of the pipe and viscosity and is inversely proportional to the fourth power of diameter:

$$
R=\frac{128 \mu l}{\rho \pi d^{4}}
$$

Ghafari et al. [9] have shown that the equivalent of capacitor in pneumatic systems is proportional to volume of the chamber containing the gas and is inversely proportional to polytropic index and temperature:

$$
C=\frac{V}{n R T}
$$

So, in case of isothermal compression, the capacitor will be linear.

It is possible to show that the equivalent of Inductor for a pipe with cross section of $A$ and length of $l$, which contains a gas with density of $\rho$, is [9]:

$$
L=\frac{\rho L}{A} l
$$

But since the density in gases is very low, we can often neglect the effect of inertia in pneumatics. The summary of

\begin{tabular}{|c|c|c|c|c|c|}
\hline Case & $\begin{array}{l}\text { Bond } \\
\text { Graph }\end{array}$ & Flow & Effort & Capacitor & Resistor \\
\hline Electrical & $\begin{array}{l}\mathrm{V} \\
\mathrm{I}\end{array}$ & I & V & $\mathrm{C}$ & $\mathrm{R}$ \\
\hline Pneumatic & $\frac{\mathrm{P}}{\dot{\mathrm{m}}}$ & $\dot{m}$ & $\mathrm{P}$ & $\frac{V}{n R T}$ & $\frac{128 \mu l}{\rho \pi d^{4}}$ \\
\hline
\end{tabular}
this analogy is shown in Table I.

TABLE I: TABLE OF EQUIVALENT VARIABLES FOR THE DIFFERENT TYPES OF SYSTEMS

\section{SIMPLE PISTON}

The analogy described in Section II was first applied to a simple compressor model. Fig. 2 illustrates such an analogy. The shaft provides a force that is converted to pressure change in the gas. This is shown by a voltage source in parallel with a variable capacitor with a ground connection. Gas can move in and out of the cylinder through leakage or valve resistors. One should note that the leak resistance in reservoir is practically infinity and is placed for the sake of correctness of the electrical circuit. The leak resistance in the cylinder is due to the fact that seals are not perfect and there is always an amount of air leaking to outside, decreasing the volumetric efficiency but the amount of the leak resistance is much higher than the valve resistance. Most of the gas will flow to a reservoir, which has a much higher fixed capacitance.

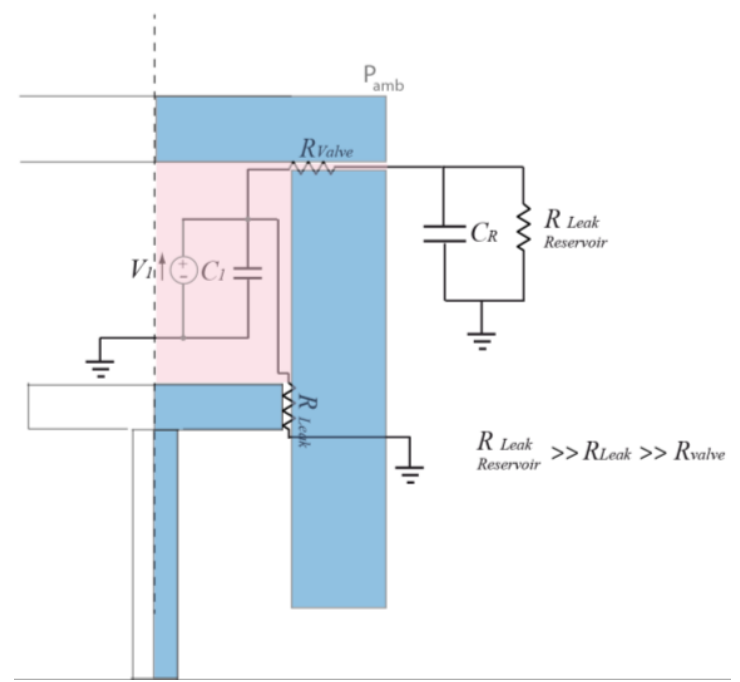

$\overline{\text { Fig. 2. Pneumatic-electric analogy for a cylinder-piston assembly. }}$

\section{FINNED PISTON}

The equivalent circuit for pneumatic-electric analogy can be found in Fig. 3. The upper chambers are connected by a small radial collecting channel that possesses resistance and capacitance effect at the same time $\left(R_{13}, C_{13}, \ldots\right)$. Also, the upper and lower chambers can exchange flow through the inner fin space (e.g., $R_{12}, R_{23}, \ldots$ ). There are also two leakage resistances, since the finned piston has two seals.

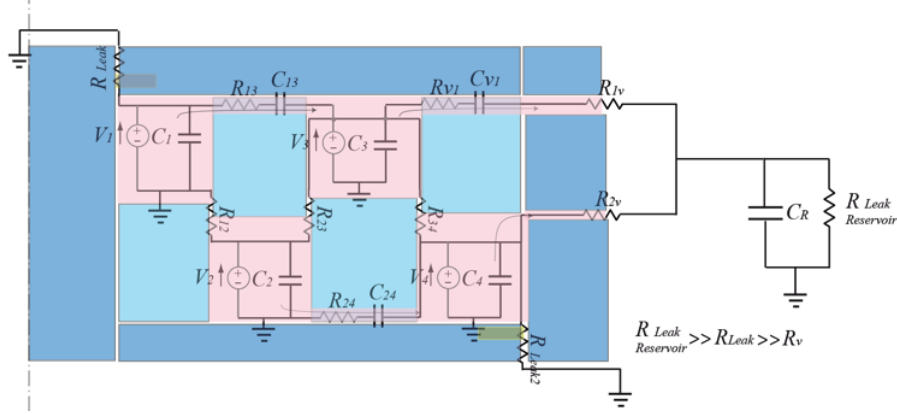

Fig. 3. Equivalent electrical circuit for pneumatic-electric analogy.

The bond graph representation of such a model can be seen in Fig. 4.

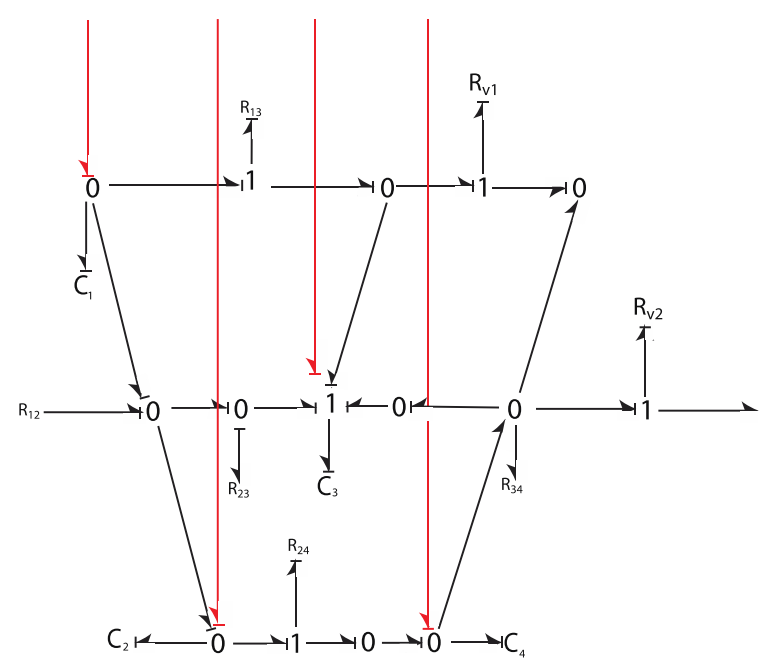

Fig. 4. Equivalent electrical circuit for pneumatic-electric analogy. 


\section{State Space Equations}

In studying complicated systems like the system described, there is an ideal opportunity to start the formulation in terms of significant physical variables and to generate simultaneous set of first-order equations. The state vector differential equations for the pneumatic part can be written as:

$$
\begin{aligned}
& \dot{X}=A X+B U ; \\
& Y=C X+D U
\end{aligned}
$$

Considering chamber's pressure as state variables, reservoir and ambient pressure as input and outgoing mass flow as the output:

$$
X=\left[\begin{array}{c}
p_{1} \\
p_{2} \\
p_{3} \\
p_{4}
\end{array}\right] ; U=\left[\begin{array}{c}
p_{a t m} \\
p_{r}
\end{array}\right] ; Y=\left[\begin{array}{c}
\dot{m}_{1 v} \\
\dot{m}_{2 v}
\end{array}\right]
$$

The state matrixes A, B, C and D can be found as:

$$
\left.\begin{array}{l}
A=\left[\begin{array}{cccc}
\frac{-1}{c_{1}}\left(\frac{1}{R_{12}}+\frac{1}{R_{13}}+\frac{1}{R_{11}}\right) & \frac{1}{c_{1}}\left(\frac{1}{R_{12}}\right) & \frac{1}{c_{1}}\left(\frac{1}{R_{13}}\right) & 0 \\
\frac{1}{c_{2}}\left(\frac{1}{R_{12}}\right) & \frac{-1}{c_{2}}\left(\frac{1}{R_{12}}+\frac{1}{R_{23}}+\frac{1}{R_{12}}\right) & \frac{1}{c_{2}}\left(\frac{1}{R_{23}}\right) & \frac{1}{c_{2}}\left(\frac{1}{R_{24}}\right) \\
\frac{1}{c_{3}}\left(\frac{1}{R_{13}}\right) & \frac{1}{c_{3}}\left(\frac{1}{R_{23}}\right) & \frac{-1}{c_{3}}\left(\frac{1}{R_{13}}+\frac{1}{R_{23}}+\frac{1}{R_{34}}+\frac{1}{R_{2 v}}\right) & \frac{1}{c_{3}}\left(\frac{1}{R_{34}}\right) \\
0 & \frac{1}{c_{4}}\left(\frac{1}{R_{24}}\right) & \frac{1}{c_{4}}\left(\frac{1}{R_{24}}\right) & \frac{-1}{c_{4}}\left(\frac{1}{R_{34}}+\frac{1}{R_{24}}+\frac{1}{R_{12}}\right)
\end{array}\right] \\
B=\left[\begin{array}{cc}
\frac{-1}{c_{1}}\left(\frac{1}{R_{l 1}}\right) & 0 \\
0 & 0 \\
0 & \frac{-1}{c_{3}}\left(\frac{1}{R_{1 v}}\right) \\
\frac{-1}{c_{4}}\left(\frac{1}{R l 2}\right) & \frac{-1}{c_{4}}\left(\frac{1}{R_{2 v}}\right)
\end{array}\right] \\
C=\left[\begin{array}{cc}
0 & \frac{-1}{c_{3}}\left(\frac{1}{R_{1 v}}\right) \\
0 & 0 \\
0 & \frac{-1}{c_{3}}\left(\frac{1}{R_{1 v}}\right) \\
0 & \frac{-1}{c_{4}}\left(\frac{1}{R_{2 v}}\right)
\end{array}\right] \\
D
\end{array}\right]
$$

\section{SiMULATION RESUlTS}

The pressure evolution comparison between a simple piston and each chamber of finned piston is shown in Fig. 5. As it can be seen, pressure evolution is less steep in the chambers of finned piston that means being closer to isothermal line.

Mass flow rate and air velocity between the second and third fins are demonstrated in comparison to inlet and outlet in Fig. 6 and Fig. 7. It is interesting to notice that however the mass flow between the fins are not high due to small pressure difference between two sides fins, but due to relatively small cross section area, the velocity of air passing through the inter-fin space is relatively high. As shown in Fig. 8 and Fig. 9, $Q d_{14}$ and $Q d_{24}$ which are inter-fin heat transfer contribute greatly to the total convection heat transfer and leads to high heat transfer rate from air to metal and high isothermal efficiency.

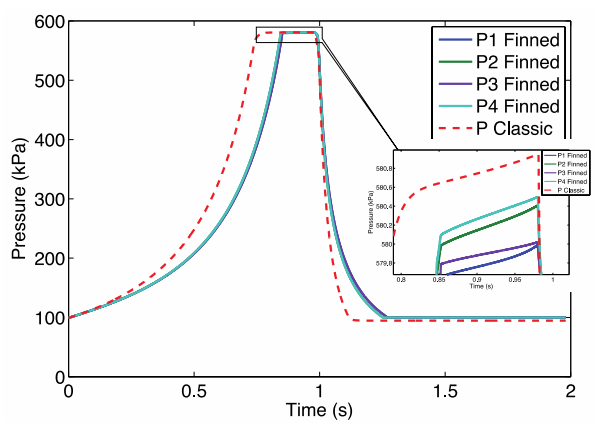

Fig. 5. Pressure evolution comparison.

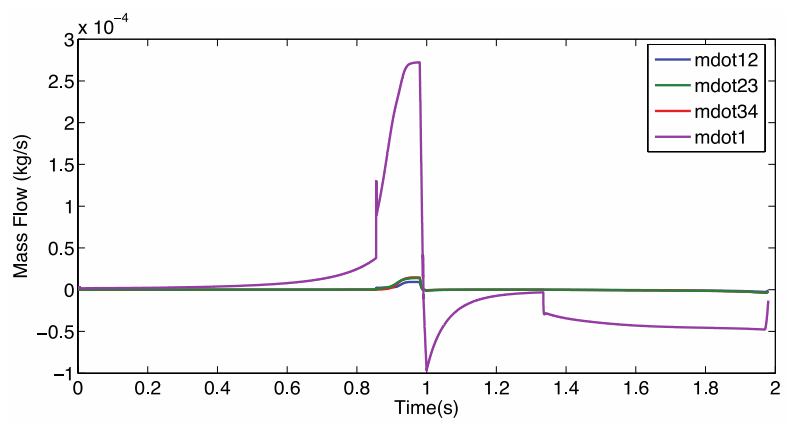

Fig. 6. Mass flow rate

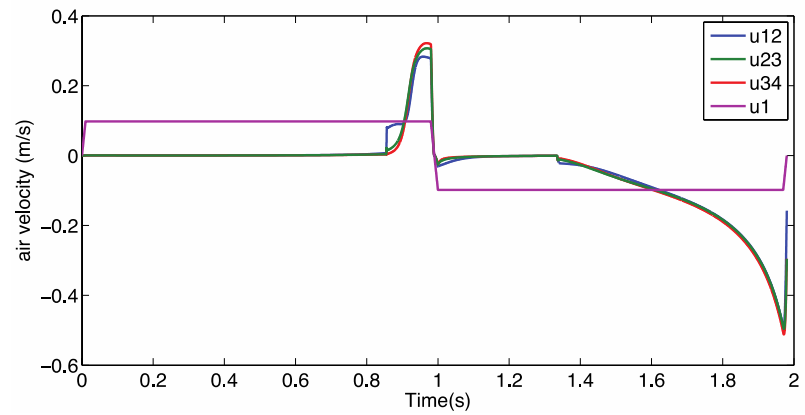

Fig. 7. Air velocity.

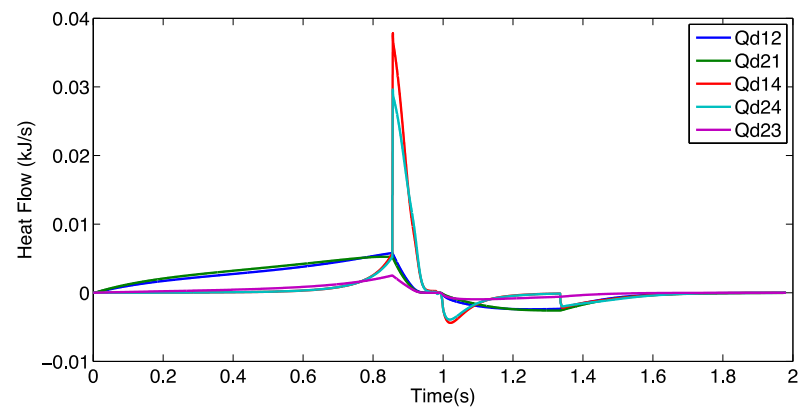

Fig. 8. Heat flow rate comparison

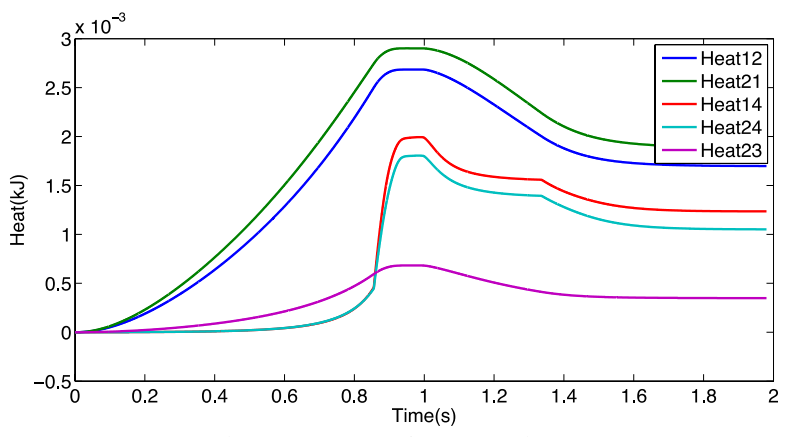

Fig. 9. Heat transfer comparison. 


\section{PNEUMATIC LOSSES}

\section{A. Inter-Fin Space}

Let us now focus on the losses associated with air flow between the inner-space fins. Fig. 10a shows such a typical gap of height about $e=100$ microns, and width of $\pi d$ where $d$ is the diameter of corresponding annuli. Also, $L$ is the length of fins, which varies along the cycle.

We suppose first that the thermal effects are negligible and the bond graph is shown by Fig. 10b. The mass flow is given in Eq. 1. And is related to volume flow as:

$$
\dot{m}=\rho \dot{V}
$$

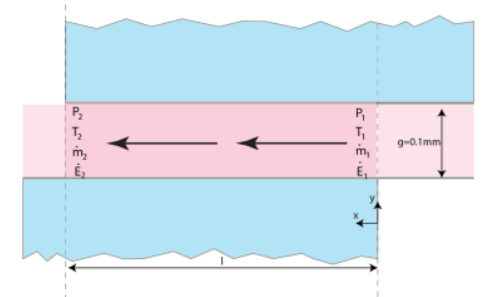

a)

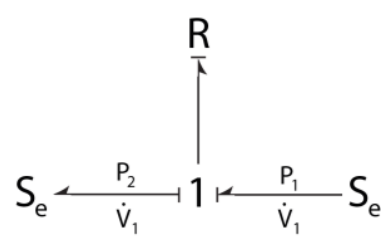

b)
Fig. 10. Pneumatic resistance with the losses of a compressible fluid.

Let us now examine the flow in the annular conduit through the same pneumatic resistance, which dissipates its energy by heating as shown in Fig. 11.

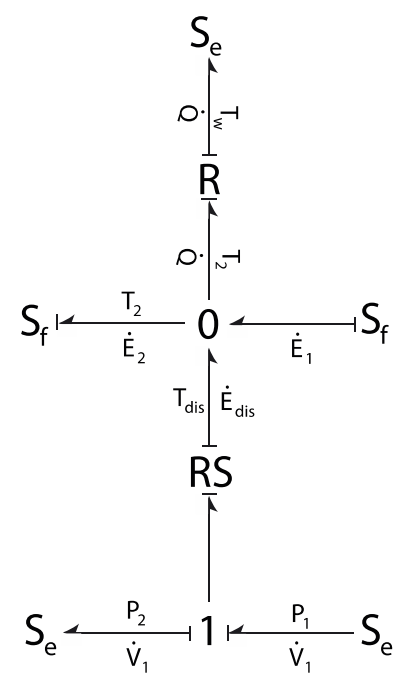

Fig. 11. Bond graph of energy dissipation in a resistance.

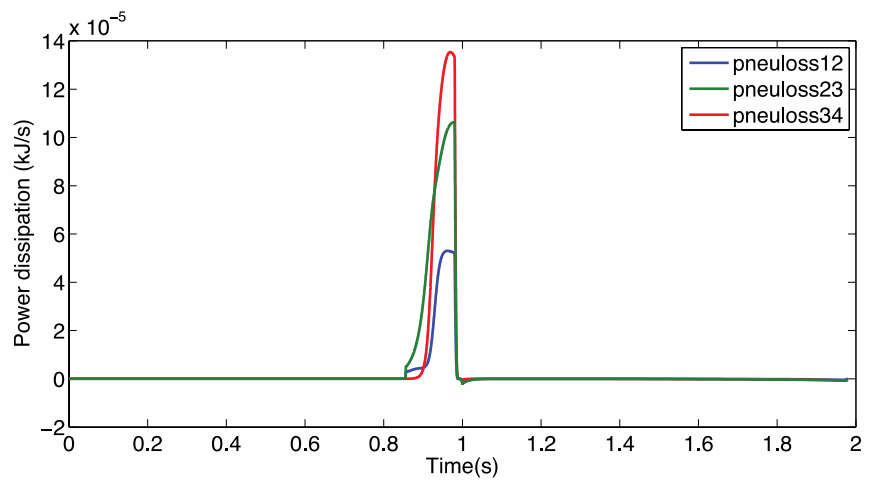

Fig. 12. Dissipated power in a the inter-fin space.

The dissipated power $p \dot{V}$ heats the air. On the bond graph the element $R$ becomes multiport $R S$ and dissipated energy $\dot{E}_{d i s}$ will be added to the internal energy of fluid $\dot{E}_{1}$. The average temperature $T_{2}$ is calculated using the constitutive equations as follows:

$$
\begin{gathered}
\dot{E}_{d i s}=\left(P_{1}-P_{2}\right) \dot{V} \\
T_{2}=\frac{1}{\dot{m} c}\left(\dot{E}_{d i s}+\dot{E}_{1}-\dot{Q}\right)
\end{gathered}
$$

The dissipated power is shown in Fig. 12.

\section{B. Valves}

The same analogy can be developed for valves. However in valves the resistance is a function of valve opening area. This parameter in turn is highly non-linear and is function of the solution of the equation of motion differential equation, which is out of the scope of this paper. Here we will only show the dissipated energy in the valves. As shown in Fig.13 and 14 , the energy dissipated in valves is about $36 \%$ of the total effective energy in the compressor.

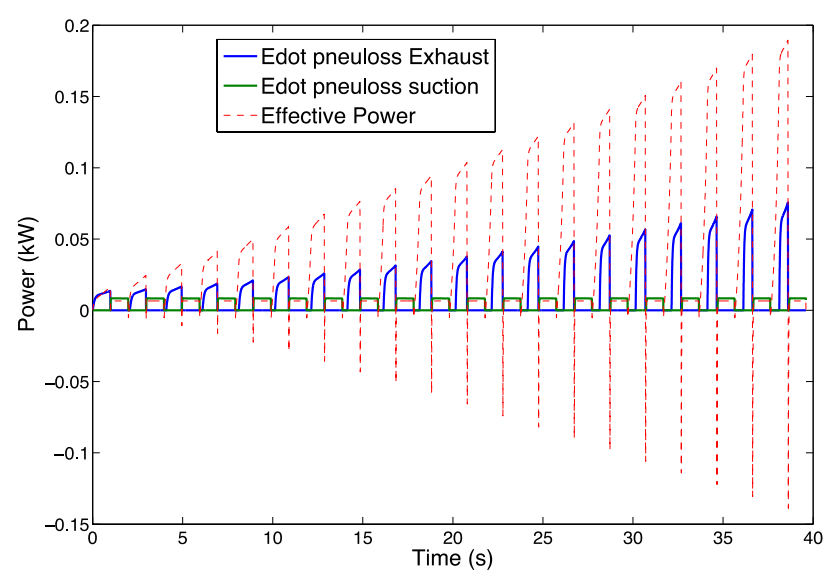

Fig. 13. Power loss in valves compared to effective power.

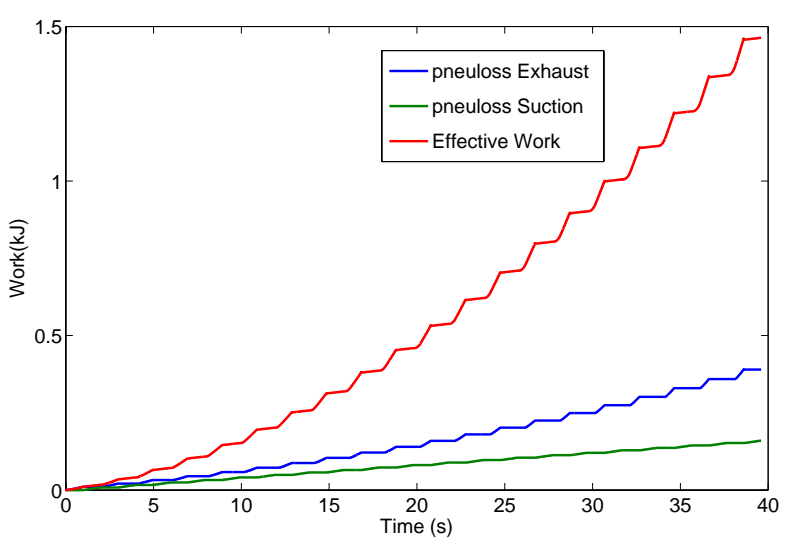

Fig. 14. Energy loss in valves compared to effective work.

\section{VELOCITY PROFILE}

It is also interesting to study the flow profile in the finned piston. In the space between two stationary fins (Fig. 15a) the flow is induced by moving piston. This kind of flow has been studied in many works [10]-[11]. In summary the flow is uniform near the piston and parabolic and fully developed at a distance of:

$$
L_{h, \text { lam }} \approx 0.05 \operatorname{Re} D
$$

For $\operatorname{Re}=32$, the hydrodynamic entry length is:

$$
L_{h, l a m} \approx 8 \mathrm{~mm}
$$


In this distance the flow is in transition mode.

But in the gap between the fins (Fig. 15b) the flow is a combination of a Couette flow and Poiseuille flow. The resulting pressure and velocity field can be found by superposition method respectively as [8]:

$$
\begin{gathered}
P=P_{0}+\frac{\partial P}{\partial x} x-\rho g z \\
u=\frac{V y}{h} x-\frac{1}{2 \mu} \frac{\partial P}{\partial x}\left(y^{2}-h y\right)
\end{gathered}
$$
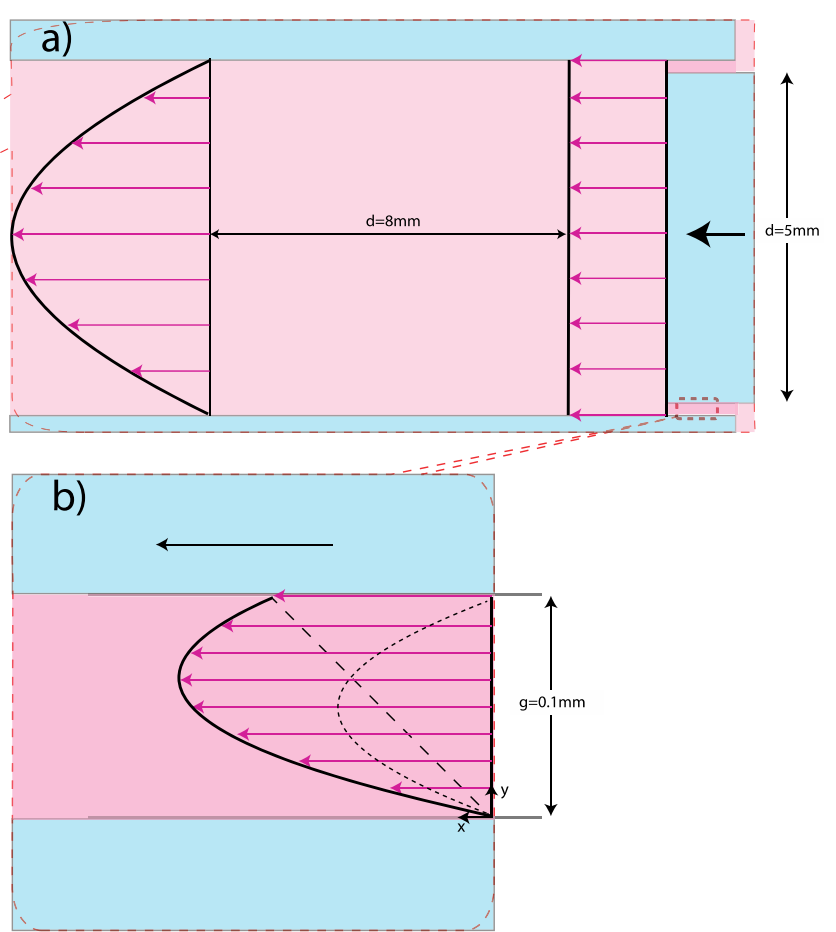

Fig. 15. Velocity profile in inter fins space.

\section{CONCLUSION}

Applying pneumatic-electricity analogy using a lumped method together with the vectorized coding to index the resistances and capacitors has made the modeling process of a complicated finned piston much easier. Also, the compression process has approached to isothermal line to a great extent thanks to increases heat transfer area.

\section{REFERENCES}

[1] L. Ljung and T. Glad, Modeling of Dynamic Systems, Prentice Hall PTR, 1st ed, 1994.

[2] J. Love, Process Automation Handbook: A Guide to Theory and Practice, Springer, 1st ed, 2007.

[3] N. F. Macia and G. J. Thaler, Modeling and Control of Dynamic Systems, Delmar Learning, 2004.

[4] K. Ogata, Modern Control Engineering, Prentice Hall, 3rd edition 1997.

[5] A. Parr, Hydraulics and Pneumatics: A Technicians and Engineers Guide, Butterworth-Heinemann, 2nd edition, 1999.

[6] M. Heidari and A. Rufer, "Modeling of a reciprocating linear compressor using energetic macroscopic representation," International Compressor Engineering Conference at Purdue, 16-19 July, 2012.

[7] M. Heidari, A. Rufer, and J. R. Thome, "Thermoelectricity analogy method for computing transient heat transfer in a new reciprocating finned piston compressor," in Proc. 15th International Heat Transfer Conference, IHTC-15, August 10-15, Kyoto, Japan, 2014.

[8] Y. A. Çengel and J. Cimbala, Essentials of Fluid Mechanics: Fundamentals and Applications, $5^{\text {th }}$ ed., McGraw-Hill, pp. 245-304, (2008).

[9] A. Ghafari, A. Maghsoudpour, and A. Pourmomtaz, Control and Dynamic Systems, $2^{\text {nd }}$ ed., K.N.Toosi University Publications (In Persian), pp. 19-72, 2006

[10] F.V. Katopodes, A. M. J. Davis, and H. A. Stone, "Piston flow in a two-dimensional channel," Physics of Fluids, vol. 12, no. 5, 1240, 2000.

[11] V. V. Meleshko and T. S. Krasnopolskaya, Piston Stokes flow in a semi-infinite channel, C. R. Acad. Sci. Paris, Série IIb, ch. 329, pp. 1-6, 2001.

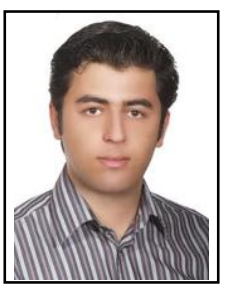

Mahbod Heidari is a PhD candidate at EPFL, Switzerland. His research interest is modeling and unsteady state analysis of complicated thermo fluidal systems. He has worked in the area energy storage and efficiency focusing on compressed air.

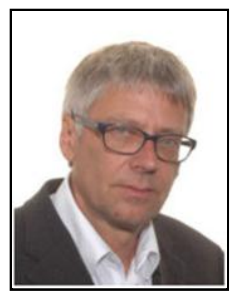

Alfred Rufer received the M.S. degree from the Ecole Polytechnique Fédérale de Lausanne (EPFL), Lausanne, Switzerland, in 1976.

In 1978, he joined ABB, Turgi, Switzerland, where he was involved in the fields of power electronics and control, such as high-power variable frequency converters for drives, and where he was a group leader involved with power electronics development beginning in 1985. In 1993, he became an assistant professor with EPFL, where, since 1996, he has been a full professor and the head of the Industrial Electronics. Laboratory (LEI).

$\mathrm{He}$ has authored or coauthored several publications on power electronics and applications. He holds several patents.

Prof. Rufer was a recipient of two IEEE Prize Paper Awards. 